\title{
Review article: irritable bowel syndrome - an evidence-based approach to diagnosis
}

\author{
B. D. $\mathrm{CASH}^{*} \&$ W. D. CHEY† \\ *Division of Gastroenterology, Uniformed Services University of the Health Sciences, Bethesda, MD, USA; †Division of \\ Gastroenterology, University of Michigan School of Medicine, Ann Arbor, MI, USA
}

Accepted for publication 8 April 2004

\section{SUMMARY}

Irritable bowel syndrome (IBS) represents one of the most common reasons for primary care visits and consultation with a gastroenterologist. It is characterized by abdominal discomfort, bloating and disturbed defecation in the absence of any identifiable physical, radiologic or laboratory abnormalities indicative of organic gastrointestinal disease. IBS is a costly disorder, responsible for significant direct and indirect costs to patients and society. Much of the cost attributed to IBS arises from the time and resources used to establish the diagnosis. Historically IBS has been viewed by many as a diagnosis of exclusion rather than as a primary diagnosis, and many patients with typical symptoms will undergo an extensive array of diagnostic tests and procedures prior to the eventual diagnosis of IBS.

Recent reviews addressing the management of such patients have cast doubt on the necessity for this degree of testing. Current best evidence does not support the routine use of blood tests, stool studies, breath tests, abdominal imaging or lower endoscopy in order to exclude organic gastrointestinal disease in patients with typical IBS symptoms without alarm features. Serological testing for celiac sprue in this population may eventually prove useful but validation of studies indicating an increased prevalence of this disease in patients with suspected IBS is needed.

The development and refinement of symptom-based criteria defining the clinical syndrome of IBS has greatly facilitated the diagnosis of this condition, which can be confidently diagnosed through the identification of typical symptoms, normal physical examination and the exclusion of alarm features. The presence of alarm features or persistent non-response to symptom-directed therapies should prompt a more detailed diagnostic evaluation dictated by the patient's predominant symptoms.

\section{INTRODUCTION}

Irritable bowel syndrome (IBS) is a chronic gastrointestinal condition characterized by abdominal discomfort, bloating and disturbed defecation. It is considered one of a group of functional gastrointestinal disorders in which a variety of factors including altered motility, abnormal visceral sensation and psychosocial factors interplay to

Correspondence to: Dr W. D. Chey, Division of Gastroenterology, University of Michigan Medical Center, 3912 Taubman Center, Ann Arbor, MI 48109-0362, USA.

E-mail: wchey@umich.edu cause symptoms. By definition, IBS is characterized by the absence of any identifiable physical, radiologic or laboratory abnormalities indicative of organic disease. IBS is a common condition, with prevalence estimates ranging between 7 and $24 \%$ in women and between 5 and $19 \%$ in men in the United States and Britain. ${ }^{1-3}$ The symptoms of IBS represent one of the most common reasons for primary care visits, and consultation with a gastroenterologist accounting for 2.4-3.5 million physician visits per year. ${ }^{1,4}$ Patients with IBS visit their physicians more frequently than those without IBS for both GI-related and non-GI-related problems, reflecting 
the increased likelihood of a variety of other conditions such as migraine headache, fibromyalgia and chronic pelvic pain. ${ }^{5,} 6$

Based upon these observations, it should come as no surprise that the annual economic consequences of IBS in the United States are substantial. It has been estimated that IBS accounts for \$1.7-10 billion in annual direct medical costs in the USA. ${ }^{7,} 8$ Perhaps more importantly, an additional \$10-20 billion in indirect costs, largely resulting from work absenteeism and decreased productivity, has been attributed to IBS. ${ }^{8,} 9$ These estimates do not include prescription or over the counter-medications for IBS, so it is likely that a substantial portion of these costs may be attributed to the diagnostic testing that is frequently conducted as part of the evaluation of patients with suspected IBS. This paper will examine the current recommendations for the diagnostic evaluation of patients with suspected IBS and will critically review the evidence regarding the yield of various diagnostic tests and procedures that are routinely performed in this group of patients in order to exclude organic disease.

\section{EVIDENCE-BASED MEDICINE APPROACH TO DIAGNOSIS}

When deciding upon the necessity of a diagnostic test, two issues deserve particular attention. First, the clinician should consider the pretest probability of the disease in question, a determination that is based upon the prevalence of that disease in patients with specific symptoms. If the pretest probability of a disease is sufficiently small, then diagnostic testing aimed at uncovering that disease is unlikely to be either clinically useful or cost-effective. Second, clinicians should consider the performance characteristics (e.g., sensitivity, specificity, positive and negative predictive values) of the diagnostic test being considered if the pretest probability of a disease is sufficiently high to warrant investigation.

The performance of a diagnostic test should shift the clinician's estimate of pretest probability of a disease up or down so that he or she may be reasonably assured that the disease being considered is either present or absent. In the case of IBS, diagnostic tests are performed in order to exclude organic diseases that may present with similar symptoms and in doing so, reassure the clinician and the patient that the diagnosis of IBS is correct. Historically, inflammatory bowel disease (IBD), colorectal cancer, systemic hormonal disturbances, enteric infections and malabsorptive diseases are of the greatest concern to the clinician faced with a patient with symptoms suggestive of IBS, and diagnostic studies designed to identify these conditions are the most commonly performed tests in patients with suspected IBS. Prevalence rates (pretest probability) for these organic gastrointestinal diseases, based on a recent systematic review of diagnostic testing in patients with suspected IBS, are presented in Table 1 along with prevalence rates of non-IBS controls. ${ }^{10}$ Though the data are limited, the prevalence of organic gastrointestinal diseases does not appear to be significantly different between patients with IBS symptoms and the normal population rates. This observation might be used by some to conclude that no diagnostic testing in patients with IBS is warranted. However, it is important to realize that these values are subject to regional and demographic variations, are based on studies with variable methodologic qualities, and are restricted to patients with IBS symptoms that are not accompanied by alarm features. Much of this paper will address these issues surrounding the diagnosis of IBS.

There are additional considerations that should be used when evaluating trials involving the performance of diagnostic tests. For details, the reader is referred to the recently published STARD initiative, which provides recommendations regarding an appropriately designed and analysed study evaluating diagnostic accuracy. ${ }^{11-13}$ The STARD initiative is designed to help editors, reviewers and readers establish the 'evidence-base' for studies about diagnosis. By adhering to specific methodologic criteria, methodologically sound studies of diagnosis should produce results that are more accurate, precise and useful to clinicians. For example, studies about the diagnostic accuracy of a test should include a

Table 1. Pretest probability of organic GI disease in patients meeting symptom-based criteria for irritable bowel syndrome (IBS)

\begin{tabular}{lll}
\hline $\begin{array}{l}\text { Organic } \\
\text { GI disease }\end{array}$ & $\begin{array}{l}\text { IBS patients } \\
\text { (pretest probability) }\end{array}$ & $\begin{array}{l}\text { General population } \\
\text { (prevalence) }(\%)\end{array}$ \\
\hline Colitis/IBD & $0.51-0.98$ & $0.3-1.2$ \\
$\begin{array}{l}\text { Colorectal cancer } \\
\text { Gastrointestinal } \\
\text { infection }\end{array}$ & $0-0.51 .7$ & $4-6$ \\
$\begin{array}{c}\text { Thyroid } \\
\text { dysfunction }\end{array}$ & 6 & N/A \\
$\begin{array}{c}\text { Lactose } \\
\text { malabsorption }\end{array}$ & $22-26$ & $5-9$ \\
\hline
\end{tabular}

Source: from Ref. ${ }^{10}$ 
blinded comparison with an established gold standard test, if available. They should also include an appropriate spectrum of patients. With respect to the diagnosis of IBS, clinical trials of diagnostic tests should include patients in whom the pretest probability of IBS or organic disease is similar. The pretest probability of any medical condition is influenced by multiple factors, such as the prevalence of the disease in question within a specific population, patient demographics and historical features, and ultimately depends upon clinical judgment. For example, a 65-year-old patient experiencing symptoms consistent with IBS who reports haematochezia has a higher pretest probability of organic gastrointestinal disease than a 25-year-old patient who has similar IBS symptoms but does not report haematochezia. Use of the STARD initiative recommendations involves assessment of the methodologic qualities of diagnostic studies and determining a quality score though application of simple flow charts and checklists. ${ }^{11}$

Patients in studies about diagnostic tests should meet prespecified inclusion and exclusion criteria and, for studies regarding the diagnosis of IBS, should fulfil the symptom-based criteria for IBS based on the Rome criteria. Investigators should be blinded to the results of the testing being performed and testing should be applied to all patients enrolled in the trial. Lastly, it is important to consider whether or not the patients enrolled in a clinical trial are similar to patients seen in routine clinical practice. This is especially important with studies of IBS because the majority of available trials regarding the diagnosis of IBS originate from tertiary care sites and such patients may not be representative of the entire spectrum of IBS patients.

\section{DIAGNOSIS OF IBS}

\section{Symptom-based criteria}

The differential diagnosis in patients with symptoms suggestive of IBS is broad and there is no reliable biological marker for the condition. As such, IBS has historically been viewed by many as a diagnosis of exclusion rather than as a primary diagnosis. In an attempt to simplify and standardize the diagnostic process for IBS, multiple symptom-based criteria (Table 2) have been developed, including the Manning, Rome I and Rome II criteria. ${ }^{14-16}$

Among the commonly used symptom-based criteria, the Manning criteria have been the most extensively

Table 2. Symptom-based criteria for the diagnosis of irritable bowel syndrome (IBS)

\begin{tabular}{|c|c|c|}
\hline Manning criteria & Rome criteria & Rome II criteria \\
\hline $\begin{array}{l}\text { Abdominal pain } \\
\text { relieved by defecation }\end{array}$ & $\begin{array}{l}\text { At least } 12 \text { weeks of continuous or } \\
\text { recurrent symptoms of the following: }\end{array}$ & $\begin{array}{l}\text { At least } 12 \text { weeks, which need not be consecutive, } \\
\text { in the preceding } 12 \text { months of abdominal discomfort } \\
\text { or pain that has two of the three features: }\end{array}$ \\
\hline $\begin{array}{l}\text { Looser stools with the } \\
\text { onset of pain }\end{array}$ & $\begin{array}{l}\text { Abdominal pain or discomfort: } \\
\text { (1) relieved with defecation, or } \\
\text { (2) associated with a change in frequency } \\
\text { of stool, or } \\
\text { (3) associated with a change in consistency } \\
\text { of stool }\end{array}$ & Relieved with defecation and/or \\
\hline $\begin{array}{l}\text { More frequent stools } \\
\text { with the onset of pain }\end{array}$ & $\begin{array}{l}\text { Two or more of the following, at least on } \\
\text { one-fourth of occasions or days: } \\
\text { (1) Altered stool frequency, or } \\
\text { (2) Altered stool form, or } \\
\text { (3) Altered stool passage, or } \\
\text { (4) Passage of mucous, or } \\
\text { (5) Bloating or feeling of abdominal } \\
\text { distention }\end{array}$ & $\begin{array}{l}\text { Onset associated with a change in frequency } \\
\text { of stool and/or }\end{array}$ \\
\hline Abdominal distension & & $\begin{array}{l}\text { Onset associated with a change in form } \\
\text { (appearance) of stool }\end{array}$ \\
\hline \multicolumn{3}{|l|}{$\begin{array}{l}\text { Passage of mucous } \\
\text { in stools }\end{array}$} \\
\hline $\begin{array}{l}\text { Sensation of incomplete } \\
\text { evacuation }\end{array}$ & & \\
\hline
\end{tabular}


evaluated. The positive predictive value (PPV) of the Manning criteria for the diagnosis of IBS has ranged between 65 and $75 \%$, depending upon the number of symptoms present and the number of symptoms included for the analysis. ${ }^{14}$ In an attempt to build upon the strengths, while limiting the weaknesses of the Manning criteria, the Rome I criteria were developed. The Rome I criteria, created through expert consensus and a careful review of the available evidence, have been less extensively studied than the Manning criteria. In one trial that evaluated the diagnostic accuracy of the Rome I criteria, the sensitivity was $65 \%$, specificity $100 \%$ and PPV 98\% in distinguishing between IBS and organic disease. ${ }^{17}$ The subsequent Rome II criteria have not been formally validated, but have been compared with the Manning and Rome I criteria in a number of studies. These studies have reported reasonably good agreement between the different criteria with kappa values of about $0.70 .^{18-20}$

It is important to note that both the Rome I and Rome II criteria were developed in order to provide a uniform framework for the selection of patients in diagnostic and therapeutic trials of IBS. In recent years, however, the extension of the Rome criteria to routine clinical practice has been encouraged. ${ }^{21,} 22$ The Rome II criteria for IBS include at least 12 weeks (which need not be consecutive), in the preceding 12 months, of abdominal discomfort or pain that is accompanied by at least two of the following three symptoms: the abdominal discomfort or pain is (a) relieved with defecation, (b) associated with a change in the frequency of defecation, and/or (c) associated with a change in the form or appearance of the stool. ${ }^{16}$ The Rome II criteria have the advantage of being easier to recall and use than the older Manning or Rome I criteria. However, recent evidence suggests that the Rome II criteria may be more restrictive than the Rome I criteria. A recent study used telephone interview data from a large, community-based sample of American women assessed the sensitivity of the Rome I and Rome II criteria. ${ }^{2}$ When the criteria were applied to over 1000 women diagnosed with IBS by their physician, Rome II was significantly less sensitive than Rome I ( $49 \%$ vs. $83 \%, P<0.001)$. This difference in sensitivity between the two sets of criteria was largely due to the more restrictive temporal pain requirement of Rome II. There was $47 \%$ agreement between Rome I and Rome II (Kappa = 0.296). This study illustrates how important seemingly small changes in the wording of symptom-based criteria can be. Further, these findings suggest that Rome II, while clearly useful for clinical research trials requiring actively symptomatic patients, likely underestimate the overall prevalence of IBS. In this way, Rome II may not be appropriate for epidemiological studies evaluating the lifetime prevalence of IBS. The role of Rome II in routine clinical practice remains poorly defined. It is clear that a percentage of patients who do not fulfil the Rome II criteria eventually are given a diagnosis of IBS, typically after exclusion of organic gastrointestinal disease following detailed diagnostic testing. Further modifications to the diagnostic criteria for IBS are expected with the forthcoming recommendations of the Rome III consensus conference.

\section{Diagnostic testing in IBS}

The Rome committee and many IBS authorities recommend that a variety of tests be considered as part of the diagnostic evaluation of patients with suspected IBS. ${ }^{15,} 16$ The most recent American Gastroenterological Association (AGA) Position Statement on IBS states that, in the absence of 'alarm features' or 'red flags' and/or any positive screening studies from referring physicians, additional diagnostic testing is not typically necessary. Red flags or alarm features include symptom onset after age 50, severe, unrelenting diarrhoea, nocturnal symptoms, unintentional weight loss, haematochezia, or a family history of organic gastrointestinal diseases such as IBD, celiac sprue or malignancy. For patients with a short duration of symptoms, demographic features such as older age at symptom onset, a family history of organic gastrointestinal disease, or a lack of concurrent psychosocial difficulties or symptom behaviours, then a complete blood count $(\mathrm{CBC})$ and occult blood (FOBT) are recommended for screening purposes. Additional tests such as erythrocyte sedimentation rate (ESR), serum chemistries, and stool examination for ova and parasites (O\&P) should be individualized, based upon symptom pattern, geographic area, and relevant clinical features such as predominant diarrhoea. Colonic visualization with flexible sigmoidoscopy, barium enema or colonoscopy (if the patient is older than 50) is also recommended. ${ }^{22}$ These evaluations are intended to rule out organic gastrointestinal diseases such as IBD, colorectal cancer and infectious diarrhoea. Other experts also recommend the use of hydrogen breath tests and thyroid function testing ${ }^{23,} 24$ to rule out lactose malabsorption and thyroid dysfunction, respectively. The degree to which these additional 
tests add to the diagnostic certainty of IBS beyond that obtained through application of the symptom-based criteria for IBS is unknown. Recent reviews addressing the management of patients with suspected IBS have cast doubt on the need for extensive routine diagnostic testing in such patients. ${ }^{10,21}$

\section{Blood tests}

Several reports ${ }^{25,26}$ have examined the use of blood tests such as $\mathrm{CBC}$, serum chemistries and thyroid function tests as part of the diagnostic evaluation of suspected IBS. In the trial by Tolliver et al., ${ }^{25} \mathrm{CBC}$ and serum chemistries were performed in 196 patients with suspected IBS. In this trial, the results of such tests failed to result in an alternative diagnosis of organic GI disease in any patient. Serum chemistries were abnormal in two patients $(1.0 \%)$ with both subjects having abnormal liver-associated enzymes. Sanders et al. $^{26}$ evaluated the use of CBC and serum chemistries in patients who fulfilled the Rome II criteria for IBS. Through these routine blood tests, they identified $6 / 300$ patients $(2.0 \%)$ with organic GI diseases. One patient was anaemic and later found to have celiac disease and two patients had abnormal liver-associated enzymes that were attributed to excess alcohol intake. Cessation or limitation of alcohol intake resulted in resolution of these laboratory abnormalities, but the effect of this intervention on IBS-like gastrointestinal symptoms was not reported. Two patients had elevated C-reactive protein levels and one had an elevated ESR. All three patients were subsequently diagnosed with IBD.

Two studies have evaluated the role of measuring thyroid-stimulating hormone (TSH) levels as part of the diagnostic evaluation of patients with suspected IBS. Hamm et al. ${ }^{27}$ performed TSH in more than 1200 patients fulfilling the Rome I criteria and identified 67 patients $(6 \%)$ with thyroid function abnormalities. These abnormalities were evenly distributed between hyper- and hypothyroidism. It is unclear whether the thyroid abnormalities identified were responsible for the patients' IBS symptoms since symptom response was not reported following correction of the thyroid dysfunction. Tolliver et al. ${ }^{25}$ identified $1 / 171 \quad(0.6 \%)$ patients with suspected IBS who had an abnormal TSH. The nature of the thyroid dysfunction was not reported, nor was the impact of therapy for the thyroid abnormality upon gastrointestinal symptoms. These data must be considered carefully, however, because thyroid function test abnormalities are common in the general population with an expected prevalence of $5-9 \% .^{28}$

Information on the yield of serological screening for celiac sprue in patients with suspected IBS has recently become available. Sanders et al. performed antigliadin antibody (IgA and $\operatorname{IgG}$ ) and endomysial antibody (EMA) testing in 300 patients with suspected IBS and in 300 age- and gender-matched asymptomatic controls in order to examine the prevalence of celiac disease in patients with IBS symptoms. Positive antibody tests were followed by upper endoscopy and distal duodenal biopsies. Sixty-six patients $(22 \%)$ with suspected IBS had positive antibody tests and 14 $(4.67 \%)$ had histologic evidence of celiac disease compared with two $(0.67 \%)$ controls. Further evidence has heightened awareness regarding the potential role of celiac disease in patients with suspected IBS. A large US study by Fasano et al. ${ }^{29}$ recently reported a prevalence of celiac disease of $1 / 133$. Prevalence rates were even higher for those with gastrointestinal symptoms (1/56) and in those with a first-degree relative with celiac disease $(1 / 22)$. In another recent study from Ireland, 30/150 (20\%) biopsy proven patients with celiac disease while only 8/162 (5\%) controls fulfilled the Rome II criteria for IBS. ${ }^{30}$ The effect of a gluten-free diet upon the gastrointestinal symptoms of patients diagnosed with celiac disease was not reported in these studies and so it remains to be proven whether or not celiac sprue was the true cause of IBS symptoms in these patients.

Conclusions from these data must be determined cautiously. Much of the data regarding the yield of blood tests in IBS patients come from tertiary care centres and clinical trials evaluating the efficacy of drug therapies. Such data may not be generalizable to the types of patients encountered in the primary or secondary care setting. On a purely pragmatic level, many patients with IBS symptoms have waited for extended periods for subspecialty evaluation and may expect some degree of testing to be performed. The degree to which patient expectations of testing and physician's insecurity about a purely symptom-based diagnosis affect the acceptance of the diagnosis of IBS and the response to therapy requires clarification. The key point to operationalizing the above data is to recognize that the application of a routine battery of blood tests in these patients is likely unnecessary. 
If blood tests are deemed necessary, their selection should be individualized based upon the specific symptoms present, their severity and the patient's concerns.

Summary of the use of blood tests for the diagnosis of IBS: Current best evidence does not support the routine use of blood tests to exclude organic gastrointestinal disease in patients who present with typical IBS symptoms without alarm features. Serological testing for celiac sprue may prove useful but validation of previous work indicating an increased prevalence in patients with suspected IBS is needed before such testing can be routinely recommended.

\section{Stool tests}

The study by Tolliver $e t$ al. ${ }^{25}$ also examined the use of FOBT in patients with suspected IBS. Fifteen out of 183 patients $(8.2 \%)$ had a positive FOBT and subsequently underwent full colonoscopic examination. Four of the 15 with positive FOBT, or $2.2 \%$ of the original cohort, had structural abnormalities identified during colonoscopy. None of these findings were felt to represent an alternative diagnosis to IBS, nor were these findings felt to provide an explanation for the patients' IBS symptoms. Examination of stool for ova and parasites is another commonly recommended test for patients with suspected IBS. Two trials have evaluated the results of stool O\&P examination in this population. ${ }^{25,}{ }^{27} \mathrm{Hamm}$ et $a .^{27}$ found that $1.7 \%(19 / 1154)$ of patients with suspected IBS had evidence of an intestinal pathogen on standard stool O\&P examination. Of these 19 subjects, eight $(0.69 \%)$ were colonized with Blastocystis hominis, a relatively common finding of unclear clinical significance. Clinical outcomes following eradication of the identified pathogens were not reported, so proof of causality remains unestablished. Likewise, Tolliver et al. $^{25}$ performed stool O\&P examinations in 170 patients with suspected IBS and found no subjects with evidence of enteric infection.

Summary of the use of stool tests for the diagnosis of IBS: Current best evidence does not support the routine use of stool tests to exclude organic gastrointestinal disease in patients who present with typical IBS symptoms without alarm features. Selected application of stool testing for O\&P and/or Giardia lamblia antigen should be considered in patients at greater risk for such infections (residing in or recent travel to areas where such infections are endemic, immunocompromised individuals).

\section{Hydrogen breath testing}

The prevalence of lactose malabsorption (typically diagnosed via abnormal hydrogen breath testing) is estimated to be approximately $25 \%$ in western countries and perhaps as high as $75 \%$ worldwide. $^{31,32}$ Several trials have reported the results of hydrogen breath testing for lactose malabsorption in patients with suspected IBS. ${ }^{25,27,33}$ One trial found that $23 \%$ $(256 / 1122)$ of patients with suspected IBS, when administered an oral 25-g lactose dose, demonstrated impaired lactose absorption. ${ }^{27}$ Response to a lactosefree diet was not reported, so it is impossible to determine how many of these subjects actually had gastrointestinal symptoms due to lactose malabsorption. In another study, 186 patients with suspected IBS were evaluated with hydrogen breath testing (50-g dose of lactose). ${ }^{25}$ These investigators found a similar prevalence of lactose malabsorption with $25.8 \%$ (48/ 186) of the cohort having abnormal results. In a subsequent publication, reflecting 3 years of follow-up, these investigators demonstrated that patients diagnosed with lactose malabsorption did not differ with respect to their gastrointestinal symptoms compared with patients without breath test evidence of lactose malabsorption. ${ }^{34}$ Bohmer and Tuynman ${ }^{35}$ were able to demonstrate a similar lactose malabsorption prevalence of $24.3 \%$ diagnosed via hydrogen breath testing in IBS patients in their study of the effects of a lactoserestricted diet on IBS symptoms. However, in contrast to the findings of Tolliver et al., these investigators did observe significant improvements in IBS symptom scores and less healthcare utilization in $75 \%$ of lactose intolerant IBS patients following education and dietary intervention over a period of 5 years. Vesa et al. ${ }^{33}$ from Finland identified lactose intolerance, via the ethanol lactose tolerance test, in $23.7 \%(101 / 427)$ of a healthy cohort. Using the Rome I criteria, $15 \%$ of lactose digesters and $15 \%$ of lactose maldigesters fulfilled criteria for IBS. While this trial was not specifically designed to evaluate the role of lactose maldigestion on IBS symptoms, the findings of comparable IBS prevalence values in lactose digesters and maldigesters suggest that lactose maldigestion did not play a significant role in the aetiology of IBS symptoms. Thus, the prevalence of lactose intolerance in patients with suspected IBS appears to be between 15 and $25 \%$, a prevalence rate very similar to that observed in the general population. Perhaps more importantly the 
clinical impact of identifying lactose intolerance in IBS patients remains unclear.

The potential role of small intestinal bacterial overgrowth (SIBO) as an aetiology for IBS symptoms has been highlighted by two recent publications. ${ }^{36,37}$ Pimentel et al. ${ }^{36}$ performed lactulose hydrogen breath testing in 202 patients fulfilling the Rome I criteria referred for evaluation of possible SIBO. Seventy-eight percent $(157 / 202)$ had breath test results that were consistent with SIBO and were treated with a 10-day course of antibiotics. Forty-seven of these patients $(29.9 \%)$ were restudied 7-10 days after completion of the antibiotics and SIBO eradication was achieved in $25(53.2 \%)$. Twelve patients $(48 \%)$ with SIBO eradication did not meet the Rome I criteria when their symptoms were reassessed (interpreted as improvement), while only four patients $(18.2 \%)$ with persistent SIBO failed to meet the Rome I criteria after treatment. While intriguing, a number of methodological limitations of the study by Pimental et al., including potential selection bias, the absence of a gold standard for the diagnosis of SIBO, the use of an unusual antibiotic to treat SIBO, short study duration and incomplete follow-up data for the majority of enrolled patients, limits the conclusions that can be drawn regarding the role of SIBO in patients with suspected IBS.

The same group of investigators subsequently reported the results of a double-blind randomized placebocontrolled trial evaluating the effects of therapy for SIBO upon IBS symptoms. ${ }^{37}$ The prevalence of SIBO in patients with IBS in this study was $84 \%$, similar to the $78 \%$ rate observed in the previous trial, and significantly greater than the $20 \%$ prevalence rate observed in healthy controls. Seven days after completion of neomycin or placebo, patients returned for symptom assessment and lactulose breath testing. IBS patients who were successfully treated for their SIBO based upon normalization of breath test results reported significantly greater improvement in IBS symptoms than patients with a persistently abnormal breath test result. There are no data evaluating the long-term effects and durability of antibiotic therapy in patients with IBS and SIBO. Further studies to address this highly controversial area are eagerly awaited.

Summary of the use of breath tests for the diagnosis of IBS: Current best evidence does not support the routine use of breath tests for lactose intolerance or SIBO to exclude organic gastrointestinal disease in patients who present with typical IBS symptoms without alarm features.

\section{Abdominal imaging}

Francis et $a .^{38}$ evaluated the role of abdominal ultrasound to identify serious abdominal or pelvic pathology in 125 patients (100 women, 25 men) with suspected IBS by the Rome I criteria. Twenty percent of women and $8 \%$ of men had some abnormality identified by abdominal ultrasound examination. Ten percent of women had pelvic abnormalities, the majority of which were gynaecological in origin. The prevalence of hepatobiliary abnormalities was similar in both women and men (10 and 8\%, respectively). Importantly, the identification of an anatomic abnormality on ultrasound did not lead to additional therapeutic measures in any patient. Further, in no cases were the authors able to correlate the abnormalities identified on ultrasound to the patients' gastrointestinal symptoms. These investigators concluded that abdominal ultrasound in patients with the 'positive diagnosis of IBS' via symptom-based criteria was not necessary and may actually be counterproductive since the identification of trivial anatomic abnormalities could conceivably lead to unnecessary patient concern and additional, more invasive tests or procedures.

Summary of the use of abdominal imaging tests for the diagnosis of IBS: Current best evidence does not support the routine use of abdominal imaging to exclude organic gastrointestinal disease in patients who present with typical IBS symptoms without alarm features.

\section{Colonic imaging}

In a small nested case-control cohort study, Lanng et al. ${ }^{39}$ performed barium enema and measured colonic transit time with radiopaque markers and plain abdominal films and found no difference in the prevalence of organic disease in patients with suspected IBS and asymptomatic controls.

Four studies have formally evaluated the yield of endoscopic investigations in patients with suspected IBS. Hamm et al. ${ }^{27}$ examined the yield of flexible sigmoidoscopy and colonoscopy in suspected IBS patients fulfilling the Rome I criteria. It is not clear from this report how many subjects underwent each individual examination. Among 306 patients studied, four $(1.3 \%)$ were given alternative diagnoses (three IBD, 
one colonic obstruction) that may have been responsible for their gastrointestinal symptoms.

Tolliver et al. $^{25}$ performed a similar analysis in 196 subjects with suspected IBS. Like the study by Hamm et al., the percentage of the cohort that underwent each examination is unclear. Forty-two colonic structural abnormalities were found in 34 subjects. Of these 42 abnormalities, two $(1.0 \%)$ patients were found to have organic gastrointestinal diseases (1 IBD, 1 cancer) that could have been potential causes of IBS symptoms. The remainder of the abnormalities consisted of benign polyps, diverticulosis, haemorrhoids, lipomata and melanosis coli.

MacIntosh et al. ${ }^{40}$ evaluated flexible sigmoidoscopy in patients with suspected IBS and in non-IBS controls. Among the IBS cohort, $89 \%$ fulfilled the Manning criteria and $84 \%$ fulfilled the Rome I criteria, while in the control group only $15 \%$ fulfilled the Manning criteria and 5\% fulfilled the Rome I criteria. No patients with suspected IBS were given alternative diagnoses to explain their gastrointestinal symptoms based upon findings at flexible sigmoidoscopy in this trial. These investigators also evaluated the diagnostic yield of performing rectal biopsies during sigmoidoscopy. No IBS patients or non-IBS controls had rectal biopsy findings that resulted in an alternative or additional diagnosis of organic GI disease.

Finally, Francis et al. ${ }^{38}$ performed colonic examination with flexible sigmoidoscopy, barium enema or colonoscopy in 125 patients who fulfilled the Rome I criteria for IBS. Except for diverticular disease that was judged to be incidental, no organic gastrointestinal disorders were identified through the performance of these tests and no patients were given alternative diagnoses to explain their gastrointestinal symptoms as a result of these examinations.

Summary of the use of colonic imaging tests for the diagnosis of IBS: Current best evidence does not support the routine use of colonic imaging to exclude organic gastrointestinal disease in patients who present with typical IBS symptoms without alarm features. Patients older than age 50 should undergo structural evaluation of the colon for the purposes of colon cancer screening.

\section{WHEN SHOULD DIAGNOSTIC TESTING BE CONSIDERED?}

The American College of Gastroenterology (ACG) Functional Gastrointestinal Disorders Task Force recently published clinical practice guidelines on the approach to the management of IBS in North America. ${ }^{21}$ Based upon the results of a recent systematic review, the task force opined that the routine performance of diagnostic tests in patients with suspected IBS without alarm features is not supported by the available literature. That is not to say that diagnostic testing never uncovers abnormal results in patients who fulfil symptom-based criteria for IBS. In fact, abnormalities are infrequently identified, but the likelihood of finding such abnormalities is not different in patients with suspected IBS when compared with non-IBS controls. The one possible exception to this statement relates to celiac disease, which in preliminary studies appears to be more prevalent in patients with suspected IBS than in non-IBS controls. The task force concluded that the current level of evidence merited a Grade C recommendation. Grade C recommendations derive from nonrandomized trials with contemporaneous controls and nonrandomized trials with historical controls.

The presence of alarm features indicates a greater pretest probability of organic disease and should not be ignored. It is, in these patients, appropriate to pursue a more aggressive diagnostic evaluation in order to exclude important organic diseases. Generally accepted 'alarm features' include new onset of symptoms in patients older than age 50, unexplained weight loss, gastrointestinal bleeding, progressive or unrelenting pain, nocturnal or large volume diarrhoea, and a family history of colon cancer, IBD or celiac sprue. Additionally, the task force recommended that any patient more than 50 years of age with IBS symptoms should undergo colonic imaging. The recommendation for colonic imaging in subjects over the age of 50 is largely predicated upon data addressing the prevalence of colon cancer. IBS symptoms in the absence of warning signs have been associated with a low likelihood of colon cancer. The average age in the studies of colonic imaging reviewed in the systematic review by Cash et al. ${ }^{10}$ ranged from 39 to 45 years and the prevalence of colorectal cancer in these studies was low, ranging from 0 to $0.51 \%$. Thus, until comparative data between IBS patients and properly gender- and age-matched controls becomes available, current recommendations for pro forma colonic imaging (for the purpose of colorectal cancer screening) in patients over the age of 50 is reasonable. Such an approach in younger patients who fulfil IBS 
symptom-based criteria and do not have warning signs is unlikely to result in a clinically meaningful rate of alternative diagnoses.

Most of the evidence relating to the importance of excluding alarm features must be inferred through indirect evidence from reports of longitudinal follow-up of patients diagnosed with IBS who did not have alarm features. In one study, Yawn et al. ${ }^{41}$ reviewed the medical records of 149 patients diagnosed with IBS. During 3 years of follow-up, one patient out of 149 $(0.67 \%)$ was diagnosed with organic disease (IBD) felt to be responsible for their gastrointestinal symptoms. Owens et $a l^{42}$ found a similar rate of alternative diagnoses in their cohort of 112 patients diagnosed with IBS. During a median follow-up of 20 years, one patient was given a subsequent alternative diagnosis that was felt to have possibly contributed to their gastrointestinal symptoms at the time of diagnosis. Harvey et $a l^{43}$ reported similar results in their study of the natural history of UK patients diagnosed with IBS by the Manning criteria followed for a period of 5 years.
Vanner et al. ${ }^{17}$ examined the relationship between the Rome criteria and the presence or absence of red flags or alarm features. They performed both retrospective and prospective analyses of patients diagnosed with IBS by the Rome I criteria over a several-year period. The investigators demonstrated that the absence of alarm features in patients who fulfilled the Rome criteria was associated with a durable diagnosis of IBS. In the retrospective arm of this trial, the sensitivity of the Rome criteria combined with the absence of alarm features was $65 \%$, the specificity was $100 \%$, and the PPV was $100 \%$ with a negative predictive value of $76 \%$. In the prospective arm of the trial, the PPV of the Rome criteria without alarm features was $98 \%$.

In patients with symptoms consistent with IBS who have alarm features, the nature and severity of a patient's symptoms will influence the choice of diagnostic testing. Most patients will undergo routine blood and stool tests depending upon their predominant symptoms. With regard to colonic imaging, it is attractive to suggest that patients with diarrhoea-
Figure 1. Evidence-based approach to the diagnosis of irritable bowel syndrome (IBS).

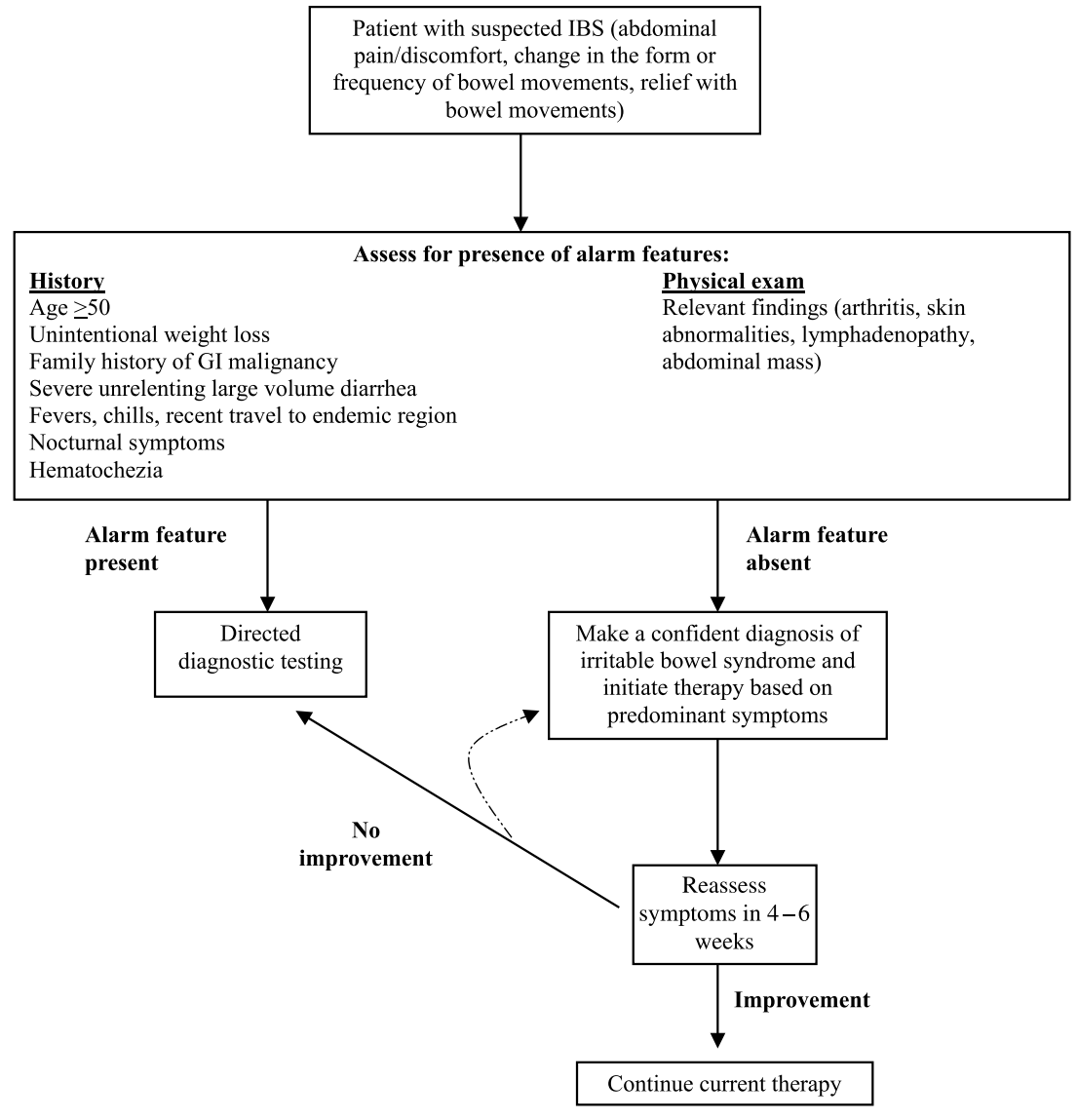


predominant symptoms undergo colonoscopy with inspection of the distal terminal ileum to exclude IBD or colon cancer. However, it is important to note that there is insufficient evidence to conclude that flexible sigmoidoscopy is not sufficient to exclude important organic disease in this population. ${ }^{44,45}$ The necessity of colonic mucosal biopsies in patients with diarrhoea-predominant symptoms also remains controversial. If a patient has clinical features suggestive of a secretory process, such as nocturnal diarrhoea, large volume diarrhoea that is unaffected by fasting or a small faecal osmotic gap $(<50 \mathrm{Osm} / \mathrm{kg})$, random colonic biopsies should be performed to exclude microscopic colitis. ${ }^{45}$ If laboratory and stool testing suggest the presence of malabsorption, upper endoscopy with small bowel biopsies may be warranted to exclude the presence of diseases such as celiac sprue.

In patients with constipation-predominant symptoms, the major objective of colonic imaging is to exclude the presence of mechanical obstruction. Colonoscopy can certainly be used for this purpose. Alternatively, particularly in younger patients where the fear of missing small lesions such as polyps is less of a concern, air contrast barium enema can effectively exclude obstructing lesions of the colon and identify colonic dilatation suggestive of pseudo-obstruction/ megacolon.

\section{CONCLUSIONS}

The development and refinement of symptom-based criteria to define the clinical syndrome of IBS has greatly facilitated the diagnosis of this common condition. IBS can be confidently diagnosed by identifying typical symptoms, performing a complete physical examination and excluding alarm features. The presence of alarm features should prompt a more detailed diagnostic evaluation directed by the patient's predominant symptoms. Current evidence does not support the performance of exhaustive testing to exclude organic diseases in patients fulfilling symptom-based IBS criteria without alarm features. Preliminary evidence suggests that celiac disease may be more prevalent in patients with suspected IBS, though these findings need to be validated in additional appropriately designed trials before routine screening for celiac disease in IBS patients can be recommended. Patients 50 years of age or greater with IBS symptoms should undergo colonoscopy (or other acceptable regimen) for age appropriate colorectal cancer screening.

If alarm features are not present and the patient fulfils symptom-based criteria, a confident diagnosis of IBS should be made and symptom-directed therapy should be initiated (Figure 1). A crucial aspect of this approach is adequate and timely follow-up. If, after appropriate therapeutic interventions, the patient reports no significant improvement, a more extensive diagnostic evaluation may be indicated. With appropriate follow-up, a clinically significant delay in the diagnosis of important organic disease is very unlikely, as demonstrated by multiple longitudinal studies that have demonstrated the durability of the diagnosis of IBS.

\section{REFERENCES}

1 Drossman DA, Whitehead WE, Camilleri M. Irritable bowel syndrome: a technical review for practice guideline development. Gastroenterology 1997; 112: 2120-37.

2 Chey WD, Olden K, Carter E, et al. Utility of the Rome I and Rome II criteria for IBS in US women. Am J Gastroenterol 2002; 97: 2803-11.

3 Sandler RS. Epidemiology of irritable bowel syndrome in the United States. Gastroenterology 1990; 99: 409-15.

4 Drossman DA, Li Z, Andruzzi E, et al. US householder survey of functional GI disorders: prevalence, sociodemography, and health impact. Dig Dis Sci 1993; 38: 156980 .

5 Whitehead WE, Palsson O, Jones KR. Systematic review of the comorbidity of irritable bowel syndrome with other disorders: what are the causes and implications? Gastroenterology 2002; 122: 1140-56.

6 Azpiroz F, Dapoigny M, Pace F, et al. Nongastrointestinal disorders in the irritable bowel syndrome. Digestion 2000; 62: 66-72.

7 Talley NJ, Gabriel SE, Harmsen WS, et al. Medical costs in community subjects with irritable bowel syndrome. Gastroenterology 1995; 109: 1736-41.

8 Goodman C, Cronin K, Gemmen E, et al. In: The Burden of Gastrointestinal Diseases. Bethesda, MD: American Gastroenterological Association, 2001: 2-65.

9 Martin R, Barron JJ, Zacker C. Irritable bowel syndrome: toward a cost-effective management approach. Am J Manag Care 2001; 7: S268-75.

10 Cash BD, Schoenfeld PS, Chey WD. The utility of diagnostic tests in irritable bowel syndrome patients: a systematic review. Am J Gastroenterol 2002; 97: 2812-9.

11 Bossuyt PM, Reitsma JB, Bruns DE, et al. Standards for reporting of diagnostic accuracy. Towards complete and accurate reporting of studies of diagnostic accuracy: the STARD initiative. Br Med J 2003; 326: 41-4.

12 Bossuyt PM, Reitsma JB, Bruns DE, et al. Towards complete and accurate reporting of studies of diagnostic accuracy: 
the STARD initiative. Standards for reporting of diagnostic accuracy. Clin Chem 2003; 49: 1-6.

13 Bossuyt PM, Reitsma JB, Bruns DE, et al. The STARD statement for reporting studies of diagnostic accuracy: explanation and elaboration. Clin Chem 2003; 49: 7-18.

14 Manning AP, Thompson WG, Heaton KW, et al. Towards a positive diagnosis of the irritable bowel syndrome. Br Med J 1978; 2: 653-4.

15 Thompson WG, Dotewall G, Drossman DA, et al. Irritable bowel syndrome: guidelines for the diagnosis. Gastroenterol Int 1989; 2: 92-5.

16 Thompson WG, Longstreth GF, Drossman DA, et al. Functional bowel disorders and functional abdominal pain. Gut 1999; 45: II43-7.

17 Vanner SJ, Depew WT, Paterson WG, et al. Predictive value of the Rome criteria for diagnosing the irritable bowel syndrome. Am J Gastrenterol 1999; 94: 2803-7.

18 Saito YA, Locke GR, Talley NJ, et al. A comparison of the Rome and Manning criteria for case identification in epidemiological investigations of irritable bowel syndrome. Am J Gastroenterol 2000; 95: 2679-81.

19 Thompson WG, Irvine EJ, Pare P, et al. Functional gastrointestinal disorders in Canada: first population-based survey using Rome II criteria with suggestions for improving the questionnaire. Dig Dis Sci 2002; 47: 225-35.

20 Mearin F, Badia X, Balboa A, et al. Irritable bowel syndrome prevalence varies enormously depending upon the employed diagnostic criteria: comparison of Rome II versus previous criteria in a general population. Scand J Gastroenterol 2001; 36: 1121-2.

21 Brandt LJ, Locke R, Olden K, et al. An evidence based approach to the diagnosis of irritable bowel syndrome in North America. Am J Gastroenterol 2002; 97: S1-S26.

22 Drossman DA, Camilleri M, Mayer EA, et al. AGA technical review on irritable bowel syndrome. Gastroenterology 2002; 123: 2108-31.

23 Schmulson MW, Chang L. Diagnostic approach to the patient with irritable bowel syndrome. Am J Med 1999; 107: 20S-6S.

24 Camilleri M. Management of the irritable bowel syndrome. Gastroenterology 2001; 120: 652-68.

25 Tolliver BA, Herrera JL, DiPalma JA. Evaluation of patients who meet clinical criteria for irritable bowel syndrome. Am J Gastroenterol 1994; 89: 176-8.

26 Sanders DS, Carter MJ, Hurlstone DP, et al. Association of adult coeliac disease with irritable bowel syndrome: a casecontrol study in patients fulfilling the Rome II criteria referred to secondary care. Lancet 2001; 358: 1504-8.

27 Hamm LR, Sorrells SC, Harding JP, et al. Additional investigations fail to alter the diagnosis of irritable bowel syndrome in subjects fulfilling the Rome criteria. Am J Gastroenterol 1999; 94: 1279-82.

28 Helfand M, Redfern CC. Screening for thyroid disease: an update. Ann Int Med 1998; 129: 144-58.
29 Fasano A, Berti I, Gerarduzzi T, et al. Prevalence of celiac disease in at-risk and not-at-risk groups in the United States: a large multicenter study. Arch Intern Med 2003; 163: 28692.

30 O'Leary C, Wieneke P, Buckley S, et al. Celiac disease and irritable bowel-type symptoms. Am J Gastroenterol 2002; 97 : 1463-7.

31 Scrimshaw NS, Murray EB. The acceptability of milk and milk products in populations with a high prevalence of lactose intolerance. Am J Clin Nutr 1988; 48: 1079-159.

32 Bourlioux P, Pochart P. Nutritional and health properties of yogurt. World Rev Nutr Diet 1988; 56: 217-58.

33 Vesa TH, Seppo LM, Marteau PR, et al. Role of irritable bowel syndrome in subjective lactose intolerance. Am J Clin Nutr 1998; 67: 710-5.

34 Tolliver BA, Jackson MS, Jackson KL, et al. Does lactose maldigestion really play a role in the irritable bowel syndrome? J Clin Gastroenterol 1996; 23: 15-7.

35 Bohmer CJ, Tuynman ARE. The effect of a lactose-restricted diet in patients with a positive lactose tolerance test, earlier diagnosed as irritable bowel syndrome: a 5-year follow-up study. Eur J Gastroenterol Hepatol 2001; 13: 941-4.

36 Pimentel M, Chow EJ, Lin HC. Eradication of small intestinal bacterial overgrowth reduces symptoms of irritable bowel syndrome. Am J Gastroenterol 2000; 95: 3503-6.

37 Pimental M, Chow EJ, Lin HC. Normalization of lactulose breath testing correlates with symptom improvement in irritable bowel syndrome: a double-blind, randomized, placebo controlled study. Am J Gastroenterol 2000; 95: 3503-6.

38 Francis CY, Duffy JN, Whorwell PJ, et al. Does routine ultrasound enhance diagnostic accuracy in irritable bowel syndrome? Am J Gastroenterol 1996; 91: 1348-50.

39 Lanng C, Mortensen D, Friis M, et al. Gastrointestinal dysfunction in a community sample of subjects with symptoms of irritable bowel syndrome. Digestion 2003; 67: 14-19.

40 MacIntosh DG, Thompson WG, Patel DG, et al. Is rectal biopsy necessary in irritable bowel syndrome? Am J Gastroenterol 1992; 87: 1407-9.

41 Yawn BP, Lydick E, Locke GR, et al. Do published guidelines for evaluation of irritable bowel syndrome reflect practice? BMC Gastroenterol 2001; 1: 11.

42 Owens DM, Nelson DK, Talley NJ. The irritable bowel syndrome: long-term prognosis and the physician-patient interaction. Ann Intern Med 1995; 122: 107-12.

43 Harvey RF, Mauad EC, Brown AM. Prognosis in the irritable bowel syndrome: a 5-year prospective study. Lancet 1987; I: 963-5.

44 Fine KD, Seidel RH, Do K. The prevalence, anatomic distribution, and diagnosis of colonic causes of chronic diarrhea. Gastrointest Endosc 2000; 51: 318-26.

45 Fine KD, Schiller LR. AGA technical review on the evaluation and management of chronic diarrhea. Gastroenterology 1999; 116: 1464-86. 